\title{
The distance to the cool T9 brown dwarf ULAS J003402.77-005206.7
}

\author{
R. L. Smart ${ }^{1}$, H. R. A. Jones ${ }^{2}$, M. G. Lattanzi ${ }^{1}$, S. K. Leggett ${ }^{3}$, S. J. Warren ${ }^{4}$, A. J. Adamson ${ }^{5}$, B. Burningham ${ }^{2}$, \\ M. Casali ${ }^{6}$, D. W. Evans ${ }^{7}$, M. J. Irwin ${ }^{7}$, and D. Pinfield ${ }^{2}$ \\ 1 Istituto Nazionale di Astrofisica, Osservatório Astronomico di Torino, Strada Osservatório 20, 10025 Pino Torinese, Italy \\ e-mail: smart@oato.inaf.it \\ 2 Centre for Astrophysics Research, Science and Technology Research Institute, University of Hertfordshire, Hatfield AL109AB, UK \\ 3 Gemini Observatory, 670 N. A'ohoku Place, Hilo, HI 96720, USA \\ 4 Imperial College London, Blackett Laboratory, Prince Consort Road, London SW7 2AZ, UK \\ 5 Joint Astronomy Centre, 660 North A'ohoku Place, Hilo, HI 96720, USA \\ ${ }^{6}$ ESO, Karl-Schwarzschild-Str. 2, 85748 Garching bei Munchen, Germany \\ 7 Institute of Astronomy, Madingley Road, Cambridge CB3 OHA, UK
}

Received 10 November 2009 / Accepted 16 December 2009

\section{ABSTRACT}

\begin{abstract}
Aims. We demonstrate the feasibility of determining parallaxes for nearby objects with the Wide Field Camera on the United Kingdom Infrared Telescope (UKIRT) using the UKIRT Infrared Deep Sky Survey as a first epoch. We determine physical parameters for ULAS J003402.77-005206.7, one of the coolest brown dwarfs currently known, using atmospheric and evolutionary models with the distance found here.

Methods. Observations over the period 10/2005 to 07/2009 were pipeline processed at the Cambridge Astronomical Survey Unit and combined to produce a parallax and proper motion using standard procedures.

Results. We determined $\pi=79.6 \pm 3.8 \mathrm{mas}, \mu_{\alpha}=-20.0 \pm 3.7 \mathrm{mas} / \mathrm{yr}$ and $\mu_{\delta}=-363.8 \pm 4.3 \mathrm{mas} / \mathrm{yr}$ for ULAS J003402.77-005206.7. Conclusions. We have made a direct parallax determination for one of the coolest objects outside of the solar system. The distance is consistent with a relatively young, 1-2 Gyr, low mass, 13-20 $M_{\mathrm{J}}$, cool, 550-600 K, brown dwarf. We present a measurement of the radial velocity that is consistent with an age between 0.5 and $4.0 \mathrm{Gyr}$.
\end{abstract}

Key words. astrometry - stars: distances - Stars: brown dwarfs - stars: fundamental parameter

\section{Introduction}

The prototype T dwarf was discovered in 1995 as a companion to the nearby M dwarf star Gl229 (Nakajima et al. 1995), and today we know of $155 \mathrm{~T}$ dwarfs (www.dwarfarchives. org as of 10/2009). The majority of these are a result of the near-infrared 2 Micron All Sky Survey (Skrutskie et al. 2006, 2MASS) and the deep optical Sloan Digital Sky Survey (York et al. 2000, SDSS). Once discovered, significant efforts were undertaken to determine their distances (Vrba et al. 2004; Tinney et al. 2003; Dahn et al. 2002) to map out the lower end of the Hertzsprung-Russell (H-R) diagram and to constrain models. However, in these surveys only a handful of objects have spectral types T7 or later and more examples of intrinsically fainter and cooler objects are crucial to understand both such objects and, in turn, exoplanets of similar masses.

In 2005 the UKIRT Infrared Deep Sky Survey (Lawrence et al. 2007, hereafter UKIDSS) began with the Wide Field Camera (Casali et al. 2007, WFCAM) large-field infrared camera. This survey is going about three magnitudes fainter than the 2MASS survey and has already revealed a number of extremely faint and cool T dwarfs (Lodieu et al. 2009a,b; Burningham et al. 2008; Pinfield et al. 2008; Chiu et al. 2008; Lodieu et al. 2007; Kendall et al. 2007). As part of the followup for the UKIDSS surveys we have started a program to determine parallaxes for the coolest objects being discovered.
The first and most obvious application of measured distances is to derive a luminosity which is used to populate the $\mathrm{H}-\mathrm{R}$ diagram and constrain models. Distances are also used to identify unresolved companions via over-luminosities, and to provide space motions which provide indications of an object's origin and age.

There is a large degree of uncertainty in current model analyses of the late-type $\mathrm{T}$ dwarfs, due to known inadequacies in the atmospheric models (e.g. Leggett et al. 2009). However, evolutionary models of cooling brown dwarfs are well understood, and show that all brown dwarfs older than about $200 \mathrm{Myr}$ have a radius within about $20 \%$ of Jupiter's (e.g. Burrows et al. 2001). Hence there is a tight relationship between luminosity and effective temperature $\left(T_{\text {eff }}\right)$ for brown dwarfs (via the Stefan-Boltzman law). Parallax determinations combined with flux-calibrated observed spectra provide luminosities, and hence the best measurements of $T_{\text {eff }}$ for brown dwarfs currently available. Once $T_{\text {eff }}$ is known, gravity and metallicity can be constrained by comparing synthetic spectra to the observations (e.g. Saumon et al. 2007). Burningham et al. (2009) use luminosity to determine $T_{\text {eff }}$ for the T8.5 Wolf 940B, and show that an analysis using synthetic spectra alone would have overestimated the temperature by $\sim 100 \mathrm{~K}$, for this $600 \mathrm{~K}$ object.

The latest spectral type currently defined is T9 and three such objects are currently known: ULAS J003402.77005206.7 (Warren et al. 2007b, hereafter ULAS 0034), 
CFBDS J005910.90-011401.3 (Delorme et al. 2008) and ULAS J133553.45 +113005.2 (Burningham et al. 2008). They have effective temperatures in the range 500 to $700 \mathrm{~K}$, and masses of 5 to $50 M_{\mathrm{J}}$ corresponding to ages of 0.1 to $10 \mathrm{Gyr}$ (e.g. Leggett et al. 2009). Here we present the first results of the UKIRT parallax program for ULAS 0034. In Sect. 2 we describe the observations and procedures for the program, in Sect. 3 we provide the results for ULAS 0034 and in Sect. 4 we find the most consistent set of physical parameters for ULAS 0034 by combining available observations and models.

\section{Observations and reduction procedures}

\subsection{Imaging}

The imaging observations were all made on the UKIRT $3.8 \mathrm{~m}$ telescope using the WFCAM imager. This is the instrument being used to carry out the UKIDSS surveys, and the calibrated images were taken directly from the pipeline for that survey (Irwin et al. 2004). All observations are carried out in queue override mode, allowing us to be very flexible in the scheduling, maximising the parallax factor and observing close to meridian passage. The observational sequence we adopt is very similar to the $J$-band imaging sequence in the Large Area Survey (LAS) component of UKIDSS (e.g. Warren et al. 2007a). A longer exposure time was used to provide an increased signal-to-noise for the targets, which are in general at the faint limit of the LAS. Specifically, we make observations in a 5 jitter (dithered) $3.2^{\prime \prime}$ cross pattern, and at each jitter position we make 4 exposures in $2 \times 2$ micro-stepped positions of 1.5 pixels, where each exposure consists of 2 co-added $10 \mathrm{~s}$ images. The total exposure time is therefore $5 \times 4 \times 2 \times 10=400 \mathrm{~s}$. In average conditions this provides a signal-to-noise of 100 at $J=18$.

ULAS 0034 was discovered in a sequence of images taken on $2005 / 10 / 04$. We use the same pointing, filter and detector setup as the $J$ image from that night in our parallax sequence. This allows us to use the discovery image as the first epoch in the parallax determination, and we reduce the time required for that determination by about one year (the time between the discovery observation and the recognition that an object warrants the investment required to determine a parallax). Nominally, to distinguish between parallactic and proper motion we aim to have observations that cover at least three years, hence the saving of one year is significant.

Parallax observations require precise astrometry. Unfortunately WFCAM has a large field-of-view with significant radial distortion, and as all four detectors are offset from the optical axis they are subject to large astrometric distortions. To minimize the differential astrometric distortion between the discovery and subsequent observations, the original pointing orientation is always used unless the object is close to the edge of a detector, in which case we move the object towards the centre. We work under the assumption that the astrometric distortion does not change for the duration of the observational program. This assumption forms the basis of all small-field, high-precision, ground-based parallax programs, even in telescopes where the focal plane is considered astrometrically flat, and is required to be true for WFCAM because of the known large astrometric distortions (Irwin et al. 2004).

During our parallax campaign WFCAM, a forward Cassegrain focus instrument, was regularly unmounted for significant periods so that the Cassegrain instruments could be used. To check that unmounting and remounting does not lead to significant changes in the astrometric map, a comparison of the

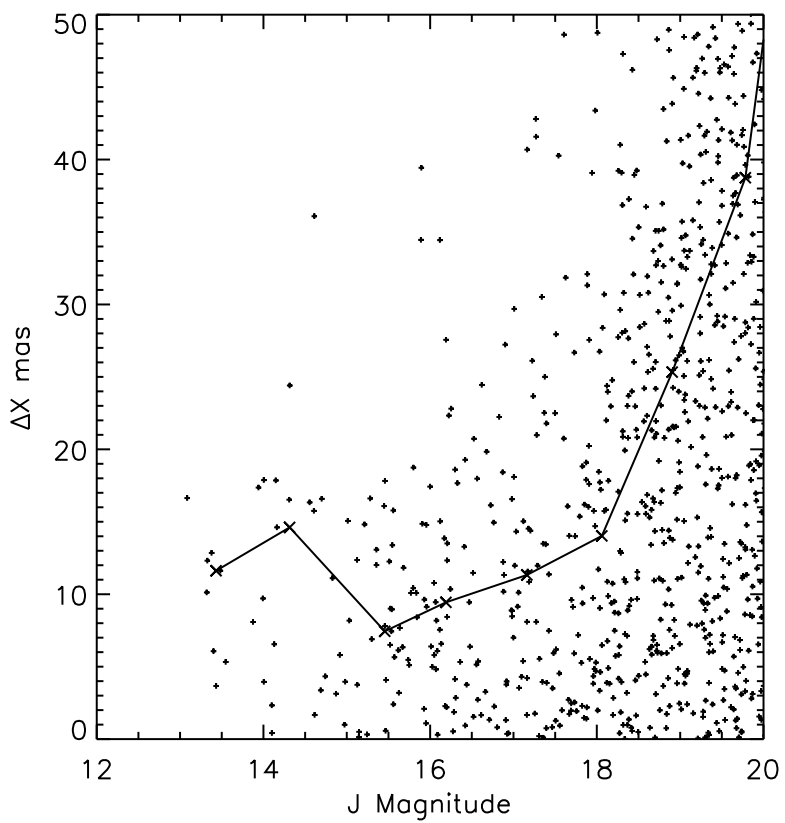

Fig. 1. Object position difference between two observations of the same cluster made on 2005/06/20 and 2005/09/04 as a function of $J$ magnitude. Plus signs are individual objects, crosses are median values.

observations of the same cluster field for the nights 2005/06/20 and 2005/09/04 were made, which straddles a period where WFCAM was unmounted and remounted. In Fig. 1 we plot the differences in local $x$ coordinates, as derived from the Cambridge Astronomical Survey Unit (CASU) pipeline discussed below. The first night was adopted as the reference system and the second night transformed using a six-constant model (translation, scale and rotation) derived from common objects. Assuming the nights were of equal quality the per observation error will be approximately equal to the combined error divided by $\sqrt{2}$. From this comparison we estimate that the per observation precision for objects to $J=18$ is around 10 mas in both coordinates including any contribution from the unmounting and remounting of WFCAM. An examination of binned residuals as a function of detector position did not reveal any systematic patterns.

The observations made with WFCAM are processed and reduced using a dedicated pipeline being run by the CASU. Since the centroid of the objects is our fundamental observation, we have extensively tested the results of this pipeline and we now describe these tests.

First we have taken the images as they come out of the CASU pipeline (i.e. dark-subtracted, flat-fielded, systematic noise removed, sky-corrected, and finally registered and stacked) and found centroids two ways: (1) fitting a two-dimensional Gaussian to the object's point source function (PSF) and (2) fitting a one-dimensional Gaussian to the object's marginal distributions above the sky background. The two-dimensional Gaussian fit is what is used in the Torino Observatory Parallax Program (Smart et al. 1999, hereafter TOPP) and the marginal distribution fit is what is suggested by Stone (1989) in the presence of a high background. We use these centroids and those from the CASU pipeline to compare eight ULAS 0034 observations over the period 2005 to 2008 , after a six-constant transform (translation, scale and rotation) to a common system. In Fig. 2 we plot the root-mean-square ( $\mathrm{rms}$ ) of the $x$ coordinate from the CASU pipeline of common stars. The median rms is 18 mas and using the other centroiding methods the rms was identical. In 


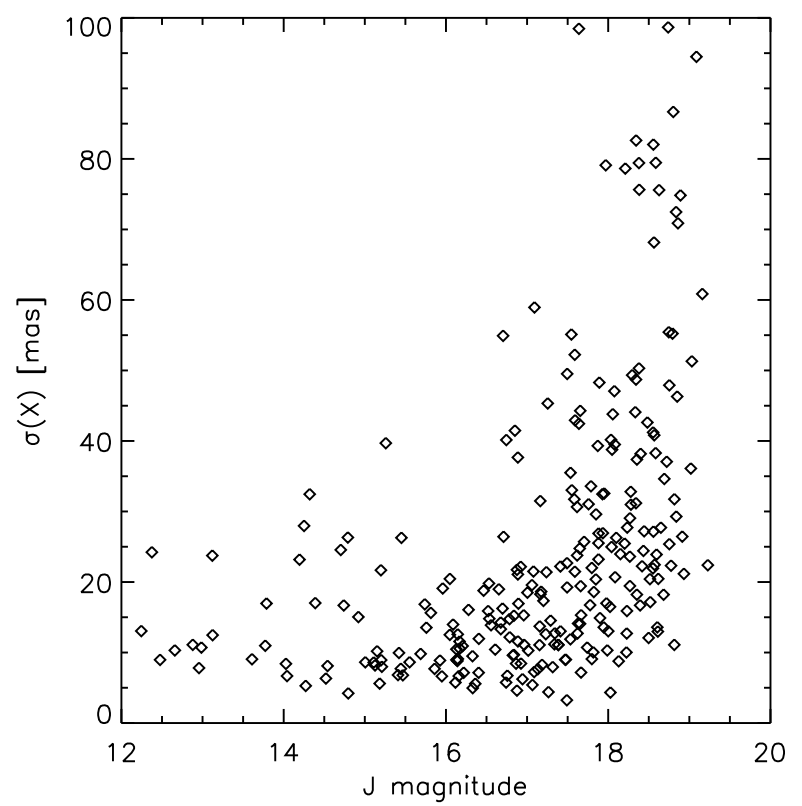

Fig. 2. Root-mean-square of the $x$ coordinates for stars in the first 8 images of the ULAS 0034 sequence which spans 2.6 years, as a function of $J$ magnitude. The centroids were all derived using the CASU pipeline.

the $y$ coordinate the median rms was slightly larger, 20 mas, but the difference between the three centroiding methods was also negligible.

We have also tried recombining the original micro-stepped images using a drizzle (Fruchter \& Hook 2002) routine rather than the CASU standard routine. In the standard routine the 4 micro-stepped images are interleaved, i.e. combined assuming a higher spatial resolution, to produce a combined image with pixel size $0.2^{\prime \prime}$ (half the $0.4^{\prime \prime}$ physical pixel size). This produces a spiky PSF as the seeing changes between the four images. The alternative approach is to combine the counts in pixels from the four images in an underlying higher resolution grid with appropriate weighting (i.e. drizzling). This gives a much smoother PSF and retains the signal-to-noise, while losing some of the resolution as adjacent pixels are correlated. Using this process caused the ULAS 0034 median rms to increase slightly from 18 to 20 mas.

As a result of these tests we have decided to use positions coming directly out of the CASU pipeline, and work under the assumption that our median error is around 18 mas. We assume the larger error found in the ULAS 0034 sequence compared to the two-epoch cluster test is due to the fact that we are comparing a wider range of observing conditions over a longer time span. We note that the centroiding errors provided by the CASU pipeline are good indications of the errors found in our test.

Once the $(x, y)$ coordinates have been determined, the parallax and proper motion of ULAS 0034 are determined using the methods adopted in the TOPP (Smart et al. 2003, 2007). We limit the reference objects used to only those within 5 arcmin of the target, as these are sufficient for a transformation, and limiting the area of the detector being modeled also limits possible differential astrometric distortion. We have made two significant changes to the procedures used in the TOPP:

1. as we are observing in the $J$-band where the atmospheric refraction is small we expect the differential reddening correction (DCR), to be negligible. This is in agreement with Tinney et al. (2003) and hence we do not apply any correction. Once we have data on more targets we will estimate the DCR and quantify the effect this could have on the target parallaxes;

2. since we have the SDSS colours in this region we use the method of Ivezić et al. (2008) to determine photometric parallaxes for the anonymous reference stars and from those we calculate the correction from relative to absolute parallax. This correction is typically less than 2 milliarcsec.

In the field there are also significant numbers of galaxies which can be used to correct from a relative to an absolute system. For the proper motions we found the mean of the galaxies was less than the error of that mean and hence, within their error, these proper motions are on an absolute system. For the parallaxes we would need to use the parameters of the galaxies as constraints (Eichhorn 1997), future work will investigate this possibility.

\subsection{Spectroscopy}

We have also obtained new near-infrared spectra of ULAS 0034 as well as of the template T8 dwarf 2MASS J0415 (Burgasser et al. 2002), for comparison. By spectral type $\mathrm{T} 8$ the $\mathrm{CH}_{4}$ and $\mathrm{H}_{2} \mathrm{O}$ molecular bands that define the $\mathrm{T}$ sequence are practically saturated. Therefore, despite the low effective temperature of ULAS $0034,550-600 \mathrm{~K}$ (see next section), relative to $750 \mathrm{~K}$ for 2MASS J0415 (Saumon et al. 2007), at low resolution the nearinfrared spectral differences are reasonably subtle. For this reason we obtained medium-resolution spectra in the $Y$ and $J$ bands to search for any spectral differences identifiable at the higher resolution. One possibility is the appearance of absorption lines due to $\mathrm{NH}_{3}$ (Leggett et al. 2007).

The observations were made with the ISAAC instrument on the ESO VLT in service mode, during several different nights within the period July to September 2008. We used a 1" slit and the medium-resolution (MR) grating, providing wavelength coverage of $0.999-1.046 \mu \mathrm{m}$ in the $Y$ band, and $1.199-1.258 \mu \mathrm{m}$ in the $J$ band. Total integration times for ULAS 0034 were $160 \mathrm{~min}$ in the $Y$ band and $120 \mathrm{~min}$ in the $J$ band, and for the much brighter source 2MASS J0415 were $40 \mathrm{~min}$ in the $Y$ band and also $40 \mathrm{~min}$ in the $J$ band. We previously obtained a spectrum with ISAAC in the $H$ band, of similar resolution, and the data reduction is described in Warren et al (2007b). We followed the same procedures for reducing the $Y$ and $J$ spectra here.

When smoothed to the lower resolution of the original GNIRS spectrum (from Warren et al. 2007b) we find the new spectra show good agreement, confirming in particular the additional absorption for ULAS 0034 compared to 2MASS J0415, in the blue wing of the $J$ band. Nevertheless a search for individual absorption lines failed to reveal any new spectral features.

The new $J$-band spectrum of ULAS 0034 is of substantially higher $\mathrm{S} / \mathrm{N}$ than the $Y$-band spectrum, and we use it here to measure the heliocentric radial velocity. The average seeing, $0.85^{\prime \prime}$, was a little less than the slit width, and determines the wavelength resolution of the spectrum, $3.5 \times 10^{-4} \mu \mathrm{m} F W H M$. The use of a slit width larger than the seeing is not ideal for the measurement of a radial velocity (which was not the primary purpose of the observations), since any mis-centering of the source in the slit will introduce a systematic error. We quantify this error in the next section, when discussing the radial velocity.

\section{Results}

In Table 1 we report the astrometric parameters derived for ULAS 0034. In Fig. 3 we plot the observations and solution derived. 
Table 1. Solution parameters for ULAS 0034 and corresponding absolute magnitudes.

\begin{tabular}{ll}
\hline \hline$\alpha, \delta \quad(\mathrm{J} 2000 . \mathrm{S})$ & $00: 34: 02.70,-00: 52: 07.7$ \\
Base epoch (yr) & 2008.1312 \\
Absolute parallax (mas) & $79.6 \pm 3.8$ \\
$\mu_{\alpha}$ (mas/yr) & $-20.0 \pm 3.7$ \\
$\mu_{\delta}$ (mas/yr) & $-363.8 \pm 4.3$ \\
Relative to absolute correction (mas) & 1.33 \\
Duration of observations, (yrs) & 3.81 \\
observations, reference stars & 17,113 \\
Absolute Magnitude in $J^{1}$ & $17.65 \pm 0.11$ \\
Absolute Magnitude in $K^{1}$ & $17.98 \pm 0.12$ \\
\hline
\end{tabular}

Notes. (1) Absolute magnitudes based on apparent magnitudes published in Warren et al. (2007b) and including both parallax and apparent magnitude errors.

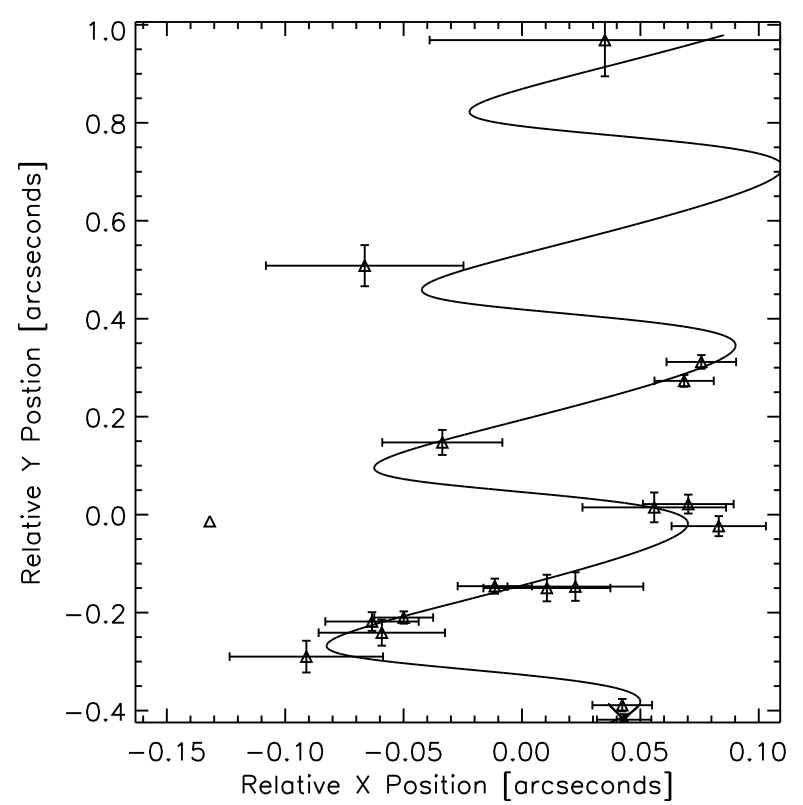

Fig. 3. Observations and solution for ULAS 0034. The point at $(0.03$, $1.0)$ is the discovery observation on $2005 / 10 / 04$ which had slightly lower signal-to-noise than subsequent observations, hence the larger error. The observation without error bars was made in twilight and in poor seeing, and is of low quality, and was rejected from the solution by three- $\sigma$ clipping.

We measured the heliocentric radial velocity of ULAS 0034 relative to 2 MASS J0415 by cross-correlating the two $J$-band spectra, finding a value $-43.6 \pm 5.4 \mathrm{~km} \mathrm{~s}^{-1}$, i.e. ULAS 0034 is blue-shifted relative to 2 MASS J0415. The quoted uncertainty accounts for the $\mathrm{S} / \mathrm{N}$ of the two spectra, but not for the systematic error, due to mis-centering of one or other object in the slit. We estimate, conservatively, that the accuracy of centering the object in the slit was as poor as $0.2^{\prime \prime}$. Then the systematic velocity error, for a single measurement, would be $\sim 10 \mathrm{~km} \mathrm{~s}^{-1}$. We computed this value by establishing the shift of the centroid of the light captured by the slit, given the measured seeing, and assuming a Gaussian profile. This error dominates the error budget for the radial velocity, and it would be possible to obtain a more accurate velocity with a different instrumental setup. Combining in quadrature the systematic error for two measurements with the random error, we obtain a total uncertainty of $15 \mathrm{~km} \mathrm{~s}^{-1}$. Taking the mean of the (much more accurate) heliocentric velocities for 2MASS J0415 given in
Table 2. Summary of physical parameters of ULAS 0034.

\begin{tabular}{ll}
\hline \hline Distance $(\mathrm{pc})$ & $12.6 \pm 0.6$ \\
$U, V, W\left(\mathrm{~km} \mathrm{~s}^{-1}\right)$ & $23 \pm 3,-8 \pm 6,-9 \pm 13$ \\
$T_{\text {eff }}(\mathrm{K})$ & $550-600$ \\
$\log g$ & 4.5 \\
Mass $\left(M_{\mathrm{J}}\right)$ & $13-20$ \\
Age $(\mathrm{Gyr})$ & $1-2$ \\
\hline
\end{tabular}

Zapatero Osorio et al. (2007), we conclude that ULAS 0034 has a radial velocity of $+7 \pm 15 \mathrm{~km} \mathrm{~s}^{-1}$. Combining this with the measured proper motions, parallax and the velocity of the Sun from Dehnen \& Binney (1998) we find a space motion, in $\mathrm{km} / \mathrm{s}$, of $U=23 \pm 3$ (radially inwards), $V=-8 \pm 6$ (in the direction of Galactic rotation), and $W=-9 \pm 13$ (vertically upwards).

Finally, we compare apparent $J$ magnitudes for our 3 year parallax sequence and find an internal standard deviation of $0.02 \mathrm{mag}$ and a systematic variation of less than 0.01 . Hence ULAS 0034 does not appear to be photometrically variable in the near-infrared.

\section{Discussion}

In the ULAS 0034 discovery paper, Warren et al. (2007b), they use near-infrared spectroscopy and mid-infrared photometry, with model atmospheres, to derive parameters of $600 \leq T_{\text {eff }} \mathrm{K} \leq$ 700 and $4.5<\log g<5.1$ for ULAS 0034, corresponding to a mass range of 15 to $36 M_{\mathrm{J}}$ and an age range of 0.5 to $8 \mathrm{Gyr}$. The inferred photometric distance is $14-22 \mathrm{pc}$. Delorme et al. (2008) use near-infrared spectra alone, with models, to estimate $T_{\text {eff }} \approx 670 \mathrm{~K}$ and $\log g \approx 4.6$ for ULAS 0034, consistent with the Warren et al. (2007b) analysis. Leggett et al. (2009, hereafter LCS09) used a model comparison to a more complete spectral energy distribution which included mid-infrared spectroscopy to constrain the physical properties of ULAS 0034 further. They find that $550 \leq T_{\text {eff }} \mathrm{K} \leq 600,4.0<\log g<4.5$ and $0.0<[\mathrm{m} / \mathrm{H}]<+0.3$. These values implied a distance of $13-16 \mathrm{pc}$, a mass of 5-20 $M_{\mathrm{J}}$ and an age of 0.1-2 Gyr. The parallax determined here allows us to further refine these results.

The measured distance of $12.6 \pm 0.6$, rules out the low gravity $(\log g=4.0)$, very low mass $\left(5-8 M_{\mathrm{J}}\right)$ and very young age (0.1-0.2 Gyr) LCS09 fits to ULAS 0034. We can check that the distance derived here is consistent with the other solutions by deriving the bolometric luminosity and comparing this to the family of $T_{\text {eff }}, \log g$ solutions calculated by structural models for a given luminosity (e.g. Saumon \& Marley 2008, their Fig. 4).

We derived the bolometric flux by interpolating between the flux calibrated $0.8-2.3 \mu \mathrm{m}$ GNIRS and $8-15 \mu \mathrm{m}$ IRS spectra presented in LCS09 using $T_{\text {eff }}=550 \mathrm{~K},[\mathrm{~m} / \mathrm{H}]=+0.3$ and $T_{\text {eff }}=600 \mathrm{~K},[\mathrm{~m} / \mathrm{H}]=0.0 \log g=4.5$ synthetic spectra. The $2.3-8 \mu \mathrm{m}$ region contributes $33-43 \%$ of the total flux. The contribution beyond $15 \mu \mathrm{m}$ was determined assuming a Rayleigh-Jeans tail to the distribution and $T_{\text {eff }}=550 \mathrm{~K}$ or $600 \mathrm{~K}$; this region contributes $\sim 8 \%$ of the total flux. The model interpolation and the Rayleigh-Jeans extrapolation were scaled by adopting the distance measured in this work, and the structural model radius of $0.11 R_{\odot}$ as per LCS09. We find a luminosity of $\log L / L_{\odot}=-5.96 \pm 0.06$, including the $4.8 \%$ uncertainty in distance and an estimated total flux uncertainty of $13 \%$. The flux uncertainty is dominated by the estimated $13 \%$ uncertainty due to the model interpolation section; other sources of error are the uncertainty in the flux calibration of the observed spectrum (3\%) 
and the assumption of a Rayleigh-Jeans tail (2\%). The luminosity is consistent with the $T_{\text {eff }}=550-600 \mathrm{~K}, \log g=4.5 \mathrm{LCS} 09$ solution for ULAS 0034, implying an age of 1-2 Gyr and a mass of 13-20 $M_{\mathrm{J}}$. Note that the measured parallax rules out the possibility that ULAS 0034 is a $550-600 \mathrm{~K}$ binary system as the distance would have to be larger than any of the LCS09 solutions. Finally we note the velocity and age are consistent with the younger populations ( $<4$ Gyr) in Figs. 5 and 6 of Holmberg et al. (2009) but outside the very young object $(<0.5 \mathrm{Gyr})$ velocity box of Fig. 6 in Zuckerman \& Song (2004).

The current UKIDSS followup program has 11 targets which will result in equally precise parallaxes by the end of 2009. Following the success for ULAS 0034 we recently added 20 other faint $\mathrm{T}$ dwarfs which will have enough observational coverage for a parallax determination by the end of 2011 .

Acknowledgements. R.S. and H.R.A.J. would like to acknowledge the support of Royal Society International Joint Project 2007/R3. This research has benefitted from the M, L, and T dwarf compendium housed at dwarfArchives.org and maintained by Chris Gelino, Davy Kirkpatrick, and Adam Burgasser. We would like to express our thanks to Luca Rizzi for scheduling help and all the observers at UKIRT who have carried out the observations used here. The United Kingdom Infrared Telescope is operated by the Joint Astronomy Centre on behalf of the Science and Technology Facilities Council of the UK SKL's research is supported by the Gemini Observatory, which is operated by the Association of Universities for Research in Astronomy, Inc., on behalf of the international Gemini partnership of Argentina, Australia, Brazil, Canada, Chile, the United Kingdom, and the United States of America. The ESO ISAAC spectra described here were obtained under programme 381.C-0036(A).

\section{References}

Burgasser, A. J., Kirkpatrick, J. D., Brown, M. E., et al. 2002, ApJ, 564, 421 Burningham, B., Pinfield, D. J., Leggett, S. K., et al. 2008, MNRAS, 391, 320 Burningham, B., Pinfield, D. J., Leggett, S. K., et al. 2009, MNRAS, 395, 1237
Burrows, A., Hubbard, W. B., Lunine, J. I., \& Liebert, J. 2001, Rev. Mod. Phys., 73,719

Casali, M., Adamson, A., Alves de Oliveira, C., et al. 2007, A\&A, 467, 777

Chiu, K., Liu, M. C., Jiang, L., et al. 2008, MNRAS, 385, L53

Dahn, C. C., Harris, H. C., Vrba, F. J., et al. 2002, AJ, 124, 1170

Dehnen, W., \& Binney, J. J. 1998, MNRAS, 298, 387

Delorme, P., Delfosse, X., Albert, L., et al. 2008, A\&A, 482, 961

Eichhorn, H. 1997, A\&A, 327, 404

Fruchter, A. S., \& Hook, R. N. 2002, PASP, 114, 144

Holmberg, J., Nordström, B., \& Andersen, J. 2009, A\&A, 501, 941 Irwin, M. J., Lewis, J., Hodgkin, S., et al. 2004, in SPIE Conf. Ser. 5493, ed. P. J. Quinn, \& A. Bridger, 411

Ivezić, Ž., Sesar, B., Jurić, M., et al. 2008, ApJ, 684, 287

Kendall, T. R., Tamura, M., Tinney, C. G., et al. 2007, A\&A, 466, 1059

Lawrence, A., Warren, S. J., Almaini, O., et al. 2007, MNRAS, 379, 1599

Leggett, S. K., Marley, M. S., Freedman, R., et al. 2007, ApJ, 667, 537

Leggett, S. K., Cushing, M. C., Saumon, D., et al. 2009, ApJ, 695, 1517

Lodieu, N., Pinfield, D. J., Leggett, S. K., et al. 2007, MNRAS, 379, 1423

Lodieu, N., Burningham, B., Hambly, N. C., \& Pinfield, D. J. 2009a, MNRAS, 397,258

Lodieu, N., Dobbie, P. D., Deacon, N. R., Venemans, B. P., \& Durant, M. 2009b, MNRAS, 395, 1631

Pinfield, D. J., Burningham, B., Tamura, M., et al. 2008, MNRAS, 390, 304

Saumon, D., \& Marley, M. S. 2008, ApJ, 689, 1327

Saumon, D., Marley, M. S., Leggett, S. K., et al. 2007, ApJ, 656, 1136

Skrutskie, M. F., Cutri, R. M., Stiening, R., et al. 2006, AJ, 131, 1163

Smart, R. L., Bucciarelli, B., Lattanzi, M. G., Massone, G., \& Chiumiento, G. 1999, A\&A, 348, 653

Smart, R. L., Lattanzi, M. G., Bucciarelli, B., et al. 2003, A\&A, 404, 317

Smart, R. L., Lattanzi, M. G., Jahreiß, H., Bucciarelli, B., \& Massone, G. 2007, A\&A, 464, 787

Stone, R. C. 1989, AJ, 97, 1227

Tinney, C. G., Burgasser, A. J., \& Kirkpatrick, J. D. 2003, AJ, 126, 975

Vrba, F. J., Henden, A. A., Luginbuhl, C. B., et al. 2004, AJ, 127, 2948

Warren, S. J., Hambly, N. C., Dye, S., et al. 2007a, MNRAS, 375, 213

Warren, S. J., Mortlock, D. J., Leggett, S. K., et al. 2007b, MNRAS, 381, 1400

York, D. G., Adelman, J., Anderson, Jr., J. E., et al. 2000, AJ, 120, 1579

Zapatero Osorio, M. R., Martín, E. L., Béjar, V. J. S., et al. 2007, ApJ, 666, 1205

Zuckerman, B., \& Song, I. 2004, ARA\&A, 42, 685 